\title{
Optimization of Delay Tracking Loops for Binary Modulated Systems
}

\author{
Meng-Hsuan Chung and Robert A. Scholtz \\ Communication Sciences Institute, \\ Electrical Engineering-Systems, \\ University of Southern California \\ email: \{menghsuc, scholtz\}@usc.edu
}

\begin{abstract}
Optimal performance of delay tracking loops in a binary linearly-modulated system is investigated, which is compared with the Cramer-Rao lower bound and the performance of a tracking loop suggested by the maximumlikelihood timing estimation.
\end{abstract}

\section{INTRODUCTION}

Synchronization is an essential part of all communication systems. The synchronization process is usually divided into two steps, namely, coarse acquisition and fine tracking. In this paper, we shall focus on the tracking part of synchronization. In [1,2], Sampaio-Neto and Scholtz addressed the problem of code-tracking loop optimization for both unmodulated and antipodally modulated DSSS systems. The optimal tracking loops they found turn out to be exactly the same as those suggested by the maximumlikelihood timing estimation (MLTE), which states that the optimal structure suggested by an open-loop estimation theory (MLTE) coincides with the optimal closed-loop tracking device.

In recent years, there has been a great interest in the research of Ultra-Wideband (UWB) systems for short-range communications or ranging applications. Among other signaling methods, binary pulse-position modulation (BPPM) is one of the possible modulation waveforms [3]. In terms of signal representation, one interesting aspect about $\mathrm{B}-\mathrm{PPM}$ is that it is not antipodal. However, for UWB systems which use pulses of nano-second durations to communicate, timing accuracy is critical to the system operations, which motivates this research on the optimization of delay tracking loops for binary modulated systems. In this paper, we shall generalize the results in [1] to an arbitrarily binary linearly-modulated system (i.e., not necessarily antipodal) and optimize the delay tracking loop for such a system. In addition, we will compare the optimal performance with the Cramer-Rao lower bound (CRLB) and that of other commonly-used tracking loops, including the one suggested by MLTE.

The received baseband waveform for a binary-modulated system on a colored additive Gaussian noise channel can be expressed as

$$
y(t)=\sqrt{P} \sum_{i} s_{b_{i}}\left(t-i T_{f}+\tau(t)\right)+n(t)
$$

This research was supported by MURI under contract DAAD1901-1-0477.

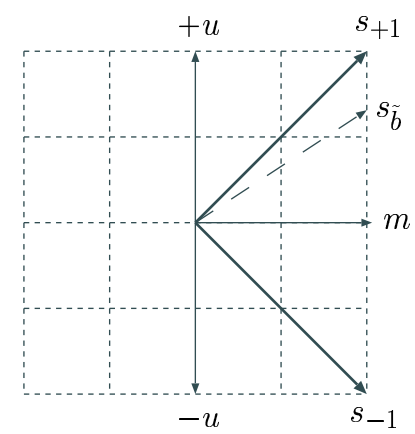

Fig. 1. Geometrical illustration of signal decomposition

where $i$ denotes the frame (or symbol) index, $P$ the average power, $b_{i} \in\{ \pm 1\}$ represents the binary data symbols, and $s_{b_{i}}(t)$ the corresponding received waveforms with unit power over one frame time $T_{f} . \tau(t)$ is the time-varying delay between the transmitter and the receiver that is to be estimated. $n(t)$ represents the possibly colored additive Gaussian noise process.

To facilitate the analysis in the following, we define the mean and modulation parts of the received noise-free signals as

$$
m(t) \triangleq \frac{1}{2}\left[s_{+1}(t)+s_{-1}(t)\right], \quad u(t) \triangleq \frac{1}{2}\left[s_{+1}(t)-s_{-1}(t)\right] .
$$

This formulation decomposes the arbitrarily binary linearly-modulated signal into a data-independent mean signal $m(t)$ and an antipodally modulated signal $u(t)$. With the definition of $m(t)$ and $u(t)$, we can write $s_{ \pm 1}(t)= \pm u(t)+m(t)$, or simply $s_{b_{i}}(t)=b_{i} u(t)+m(t)$. Also, when $\left\|s_{+1}\right\|=\left\|s_{-1}\right\|$, the mean and modulation parts are orthogonal, i.e., $\langle m, u\rangle=0$. This is illustrated geometrically in Fig. 1. Within this general signal model for binary modulations, we have two special cases: (i) when the signal is unmodulated, $u(t)=0$; (ii) when the signal is antipodally modulated, $m(t)=0$.

\section{Maximum-Likelihood Timing Estimation}

In this section, we first consider the maximum-likelihood timing estimation of the delay $\tau$ over AWGN channel and assume $\tau$ is a constant for the time being. Then we derive a closed-loop implementation suggested by it for binary modulated systems. Suppose we have observations of $y(t)$ over $N$ frames. Following the approach in [2] and using 
the signal decomposition in (2), the log-likelihood function of $\tau$ averaged over the random data $b_{i}$ with equal prior probabilities is given by

$$
\begin{aligned}
L(y, \tau) & =\sum_{i=0}^{N-1} \frac{2 \sqrt{P}}{N_{0}} \int_{0}^{T_{f}} y\left(t+i T_{f}\right) m(t-\tau) d t \\
& +\sum_{i=0}^{N-1} \ln \cosh \left\{\frac{2 \sqrt{P}}{N_{0}} \int_{0}^{T_{f}} y\left(t+i T_{f}\right) u(t-\tau) d t\right\}
\end{aligned}
$$

where the terms independent of $\tau$ have been dropped. The ML estimate of $\tau$ is the value that maximizes (3). MLTE leads to an open-loop structure which tests different possible values of $\tau$ and determine the ML estimate $\hat{\tau}_{M L}$.

Comparing (3) with previous results in [2], the first and the second terms in (3) correspond to the mean signal $m(t)$ and the modulation part $u(t)$, respectively. This simple formulation of the average log-likelihood function for an arbitrarily binary-modulated system is possible only if we use the signal decomposition in (2).

A necessary condition for ML estimate $\hat{\tau}$ is that $\dot{L}(r, \hat{\tau})=0$ where $\dot{L}$ denotes the derivative of $L$ with respect to $\tau$. The derivative of the log-likelihood function is given by

$$
\begin{aligned}
\dot{L}(y, \tau) & =\sum_{i=0}^{N-1} \int_{0}^{T_{f}} y\left(t+i T_{f}\right)\left[\dot{m}(t-\tau)+\tilde{b}_{i} \dot{u}(t-\tau)\right] d t \\
& =\sum_{i=0}^{N-1} \int_{0}^{T_{f}} y\left(t+i T_{f}\right) \dot{s}_{\tilde{b}_{i}}(t-\tau) d t
\end{aligned}
$$

where $s_{\tilde{b}_{i}}(t)=m(t)+\tilde{b}_{i} u(t)$ and

$$
\tilde{b}_{i} \triangleq \tanh \left\{\frac{2 \sqrt{P}}{N_{0}} \int_{0}^{T_{f}} y\left(t+i T_{f}\right) u(t-\tau) d t\right\} .
$$

The argument of $\tanh (\cdot)$ in (5) is actually the matched-filter output for binary data detections. Also, $|\tanh (\cdot)| \leq 1$. Hence the MLTE is interpreted to first make a soft decision about $b_{i}$ and then remove the effect of random data by multiplying the soft decision back to the modulation part $u(t)$.

Observing (4) indicates that the MLTE suggests using the derivative of the incoming noise-free signal as the local reference waveform to perform cross-correlation, then driving this quantity to zero in order to obtain an estimate of $\tau$. If the signal is modulated, soft decision should be made first to remove the modulation and then synthesize the reference waveform.

Note that in general the estimated signal $s_{\tilde{b}_{i}}$ is not in the signal alphabet, i.e., $s_{\tilde{b}_{i}} \notin\left\{s_{+1}, s_{-1}\right\}$ as shown in Fig. 1, which may impose some difficulties in implementation of the MLTE. A common practice is to use high SNR approximation by making $\tanh (x) \approx \operatorname{sgn}(x)$ [5] or low SNR approximation $\tanh (x) \approx x[1]$. Observations about (5) also indicate that the MLTE suggested structure explicitly depends on $\sqrt{P} / N_{0}$.

Although the MLTE gives the optimum open-loop solution, the closed-loop structure suggested by it may not

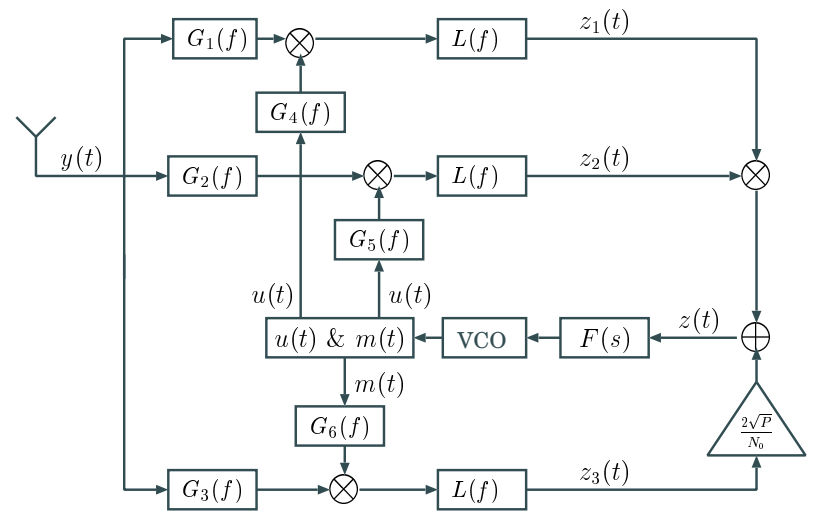

Fig. 2. The tracking loop suggested by MLTE with pre-correlation filters

be optimal among all closed-loop tracking devices. In the following section, we will consider the tracking loop optimization in the framework of filter optimization and see how well a tracking loop can perform compared with the Cramer-Rao lower bound.

\section{Filter Optimization for Tracking Loops}

In [1], Sampaio-Neto and Scholtz cast the problem of tracking loop optimization in the framework of precorrelation filter design. Here, we continue their effort to find the optimal tracking loop for a binary linearlymodulated (not necessarily antipodal) system over colored Gaussian noise channel. From (4) and following the approach in [2] we are able to obtain a closed-loop structure which approximates the MLTE at low SNR. The derived tracking loop is shown in Fig. 2 which consists of linear precorrelation filters $G_{i}(f)$ 's. The lower branch corresponds to the mean signal $m(t)$-related terms in (4), while the upper and middle branches correspond to the modulation part $u(t)$-related terms, which includes the soft-decision signal synthesis. $G_{1}(f), G_{2}(f)$, and $G_{3}(f)$ filter the received signal $y(t)$ and then the filtered signals are correlated with locally generated references consisting of $u(t)$ and $m(t)$ shaped by $G_{4}(f), G_{5}(f)$, and $G_{6}(f)$. These filters replace the possible differentiators in (4) and are to be optimized to obtain optimal tracking performance. With the addition of these filters in the loop, we are also able to deal with colored interference which the loop without the filters was not derived for. Also, the integration devices have been replaced by low-pass filters $L(f)$ with bandwidth roughly equal to the frame rate. Furthermore, we have replaced $\tanh \left(\frac{2 \sqrt{P}}{N_{0}} x\right)$ in (5) simply by $x$. The unit disagreement due to the omission of $\frac{2 \sqrt{P}}{N_{0}}$ is compensated for by the amplifier with gain $\frac{2 \sqrt{P}}{N_{0}}$ on the lower branch. Also, $F(s)$ denotes the low-pass loop filter and VCO controls the timing of $m(t)$ and $u(t)$.

To ensure that the S-curve of the loop in Fig. 2 is an odd function of the timing error and that the noise process in the loop has zero mean, we impose the following conditions on the filters $G_{i}(f)$ 's: 
- $G_{1}^{*}(f) G_{4}(f)$ is real and even for all $\mathrm{f}$.

- $G_{2}^{*}(f) G_{5}(f)$ is imaginary and odd for all $\mathrm{f}$.

- $G_{3}^{*}(f) G_{6}(f)$ is imaginary and odd for all $\mathrm{f}$.

Before we proceed, we define some useful functionals which make the analysis more compact.

$$
\begin{aligned}
& \Gamma_{1}(f) \triangleq G_{1}^{*}(f) G_{4}(f) \sqrt{S_{n}(f) E_{u}(f)} \\
& \Gamma_{2}(f) \triangleq G_{2}^{*}(f) G_{5}(f) \sqrt{S_{n}(f) E_{u}(f)} \\
& \Gamma_{3}(f) \triangleq G_{3}^{*}(f) G_{6}(f) \sqrt{S_{n}(f) E_{m}(f)} \\
& A(f) \triangleq \sqrt{\frac{E_{u}(f)}{S_{n}(f)}}, \quad A_{p}(f) \triangleq-j 2 \pi f \tau_{m} A(f) \\
& B(f) \triangleq \frac{E_{m u}^{*}(f)}{\sqrt{S_{n}(f) E_{u}(f)}}, \quad B_{p}(f) \triangleq-j 2 \pi f \tau_{m} B(f) \\
& C(f) \triangleq \sqrt{\frac{E_{m}(f)}{S_{n}(f)}}, C_{p}(f) \triangleq-j 2 \pi f \tau_{m} C(f)
\end{aligned}
$$

where $S_{n}(f)$ is the PSD of the colored Gaussian noise $n(t)$. $E_{m}(f)$ and $E_{u}(f)$ are the energy spectral densities of $m(t)$ and $u(t)$, respectively, while $E_{m u}(f)$ denotes their crossenergy spectral density. $\tau_{m}$ is a normalization constant with unit second to make the results scalable.

We now define $\epsilon \triangleq(\tau-\hat{\tau}) / \tau_{m}$ as the normalized timing estimation error. In linear analysis of tracking theory [4], the two most important quantities are: (i) $s^{\prime}(\epsilon=0)$ : the derivative of the mean signal of $z(t)$ (the S-curve) with respect to $\epsilon$ at $\epsilon=0$; and (ii) $S_{n_{e}}(f=0, \epsilon=0)$ : the PSD of the noise (mean-free) component of $z(t)$ at frequency zero $(f=0)$ and zero estimation error $(\epsilon=0)$. We then define $N_{\text {eff }}$, the effective noise PSD (at D.C.) in the loop, as follows.

$$
N_{\text {eff }} \triangleq \frac{S_{n_{e}}(0,0)}{\left[s^{\prime}(0)\right]^{2}}
$$

After lengthy derivations which are omitted here, these two quantities are given by

$$
\begin{aligned}
s^{\prime}(0)= & \frac{2 P}{T_{f} N_{0}}<\Gamma_{3}, C_{p}>+\frac{\alpha P}{T_{f}{ }^{2}}<\Gamma_{1}, A><\Gamma_{2}, A_{p}> \\
& +\frac{P}{T_{f}^{2}}<\Gamma_{1}, B_{p}><\Gamma_{2}, B> \\
S_{n_{e}}(0,0)= & \frac{4 P}{T_{f} N_{0}^{2}}<\Gamma_{3}, \Gamma_{3}>+\frac{\alpha^{\prime} P}{T_{f}{ }^{3}}<\Gamma_{1}, A>^{2}<\Gamma_{2}, \Gamma_{2}> \\
& +\frac{\beta^{\prime}}{T_{f}{ }^{3}}<\Gamma_{1}, \Gamma_{1}><\Gamma_{2}, \Gamma_{2}> \\
& +\frac{4 P}{T_{f}^{2} N_{0}}<\Gamma_{2}, B><\Gamma_{3} \frac{B}{C}, \Gamma_{1}> \\
& +\frac{P}{T_{f}{ }^{3}}<\Gamma_{2}, B>^{2}<\Gamma_{1}, \Gamma_{1}>
\end{aligned}
$$

where

$$
\begin{aligned}
\alpha & \triangleq \int_{-\infty}^{\infty} S_{d}(f)|L(f)|^{2} d f \leq 1, \\
\alpha^{\prime} & \triangleq \int_{-\infty}^{\infty} S_{d}(f)|L(f)|^{4} d f \leq 1, \\
\beta^{\prime} & \triangleq T_{f} \int_{-\infty}^{\infty}|L(f)|^{4} d f .
\end{aligned}
$$

and $S_{d}(f)$ is the PSD of the NRZ data sequence $\sum_{i=-\infty}^{+\infty} b_{i} \Pi\left(t-i T_{f}\right)$ with $\Pi(t)$ being the rectangle function with duration $T_{f}$.
The timing error variance $\sigma_{\epsilon}^{2} \triangleq E\left[(\tau-\hat{\tau})^{2} / \tau_{m}^{2}\right]$ of the tracking loop is equal to $2 B_{L} N_{\text {eff }}$ where $B_{L}$ is the equivalent one-sided loop bandwidth. Therefore, to minimize the timing error is equivalent to minimize $N_{\text {eff }}$ through optimizations of $\Gamma_{i}(f)$ 's (and hence $G_{i}(f)$ 's). Before we start to optimize these pre-correlation filters, let's look at two special cases which have been considered in $[1,2]$, namely, the unmodulated and anti-podally modulated systems.

\section{A. Unmodulated System}

When the signal is not modulated, we have $u(t)=0$. The effective noise PSD (7) can be simplified by eliminating all terms involving $u(t)$. The result is given in (10) and can be minimized using Schwartz' inequality as follows.

$$
N_{e f f}=\frac{\frac{4 P}{T_{f} N_{0}^{2}}<\Gamma_{3}, \Gamma_{3}>}{\left[\frac{2 P}{T_{f} N_{0}}<\Gamma_{3}, C_{p}>\right]^{2}} \geq \frac{T_{f}}{P<C_{p}, C_{p}>}
$$

where equality holds when $\Gamma_{3}=k_{3} C_{p}$ and $k_{3}$ is an arbitrary positive constant; that is when $G_{3}^{*}(f) G_{6}(f)=$ $-k_{1} j 2 \pi f \tau_{m} / S_{n}(f)$, which means we should put a whitening filter in front to whiten the colored noise process and use a differentiator to generate the derivative of the noise-free signal as the local reference. Therefore, the optimal filters in AWGN are those suggested by the MLTE and hence the MLTE gives the optimal delay tracking loop among all closed-loop tracking devices.

\section{B. Antipodally Modulated System}

When the signal is antipodally modulated, we have $m(t)=0$. Similarly, the $N_{\text {eff }}$ in the case can be derived by eliminating all terms involving $m(t)$ in (7). The result is given below and again using Schwartz' inequality we can minimize $N_{\text {eff }}$ easily.

$$
\begin{aligned}
N_{e f f} & =\frac{\frac{\alpha^{\prime} P}{T_{f}{ }^{3}}<\Gamma_{1}, A>^{2}<\Gamma_{2}, \Gamma_{2}>+\frac{\beta^{\prime}}{T_{f}{ }^{3}}<\Gamma_{1}, \Gamma_{1}><\Gamma_{2}, \Gamma_{2}>}{\left[\frac{\alpha P}{T_{f}{ }^{2}}<\Gamma_{1}, A><\Gamma_{2}, A_{p}>\right]^{2}} \\
& \geq \frac{1}{<A_{p}, A_{p}>} \frac{\alpha^{\prime} T_{f}}{\alpha^{2} P}+\frac{\beta^{\prime} T_{f}}{\alpha^{2} P^{2}} \frac{1}{<A, A>}
\end{aligned}
$$

where equality holds when $\Gamma_{1}=k_{1} A$ and $\Gamma_{2}=k_{2} A_{p}$ and $k_{1}$ and $k_{2}$ are arbitrary positive constants; that is when $G_{1}^{*}(f) G_{4}(f)=k_{1} 1 / S_{n}(f)$ and $G_{2}^{*}(f) G_{5}(f)=$ $-k_{2} j 2 \pi f \tau_{m} / S_{n}(f)$, which means we should set $G_{1}^{*}(f) G_{4}(\mathrm{f})$ as a whitening filter and make a soft decision (small SNR approximation) about $b_{i}$ and using $G_{2}^{*}(f) G_{5}(f)$ to whiten and differentiate the signal to generate the local reference. Again, MLTE suggests the optimal delay tracking loop in AWGN among all closed-loop tracking devices for an antipodally modulated system.

\section{Arbitrarily Binary-Modulated System}

We now consider a more general case when the signal is arbitrarily binary-modulated, that is when neither $m(t)$ nor $u(t)$ is zero. The effective noise spectrum $N_{e f f}$ is given in (12). The first and second terms in the denominator correspond to the slope of the S-curve at $\epsilon=0$ in the unmodulated and antipodally modulated cases, while the third 


$$
N_{e f f}=\frac{\frac{4 P}{T_{f} N_{0}{ }^{2}}<\Gamma_{3}, \Gamma_{3}>+\frac{\alpha^{\prime} P}{T_{f}{ }^{3}}<\Gamma_{1}, A>^{2}<\Gamma_{2}, \Gamma_{2}>+\frac{\beta^{\prime}}{T_{f}{ }^{3}}<\Gamma_{1}, \Gamma_{1}><\Gamma_{2}, \Gamma_{2}>+\frac{4 P}{T_{f}{ }^{2} N_{0}}<\Gamma_{2}, B><\Gamma_{3} \frac{B}{C}, \Gamma_{1}>+\frac{P}{T_{f}{ }^{3}}<\Gamma_{2}, B>2<\Gamma_{1}, \Gamma_{1}>}{\left[\frac{2 P}{T_{f} N_{0}}<\Gamma_{3}, C_{p}>+\frac{\alpha P}{T_{f}{ }^{2}}<\Gamma_{1}, A><\Gamma_{2}, A_{p}>+\frac{P}{T_{f}{ }^{2}}<\Gamma_{1}, B p><\Gamma_{2}, B>\right]^{2}}
$$

term results from the cross-correlation between $m(t)$ and $u(t)$ related terms. Similarly in the numerator, the first term corresponds to the noise PSD at $f=0$ and $\epsilon=0$ in the unmodulated case, while the second and third terms the anti-podally modulated case. The rest (fourth and fifth terms) comes from the cross-correlation between them.

In the following analysis, we will not show the dependence of functionals on frequency $f$ for conciseness. To find the optimal pre-correlation filters $G_{i}$ 's, we first resolve $\Gamma_{i}$ 's into components as follows.

$$
\begin{aligned}
& \Gamma_{1}=g_{1} A+g_{2} B_{p}+D \\
& \Gamma_{2}=g_{3} A_{p}+g_{4} B+E \\
& \Gamma_{3}=g_{5} C_{p}+F
\end{aligned}
$$

$\Gamma_{1}$ is resolved into components on a non-orthogonal basis $\left\{A, B_{p}\right\}$ and $D$ is the part of $\Gamma_{1}$ that is orthogonal to $A$ and $B_{p} . g_{1}$ and $g_{2}$ denote the projections of $\Gamma_{1}$ onto them. Similarly for $\Gamma_{2}, E$ is the part of $\Gamma_{2}$ that is orthogonal to $A_{p}$ and $B$. Also, $F$ is the part of $\Gamma_{3}$ that is orthogonal to $C_{p}$. Therefore, to find the optimal $\Gamma_{i}$ 's, we have to optimize $D, E, F$, and $g_{i}$ 's.

To minimize $N_{e f f}$, we begin by finding the optimal $E$. We notice that $E$ only appears in the second and the third terms of the denominator in (12). After grouping together terms involving $E$ and discarding the rest, we have an object function $J(E)$ to minimize, which is given by

$$
J(E)=\left[\frac{\alpha^{\prime} P}{T_{f}^{3}}<\Gamma_{1}, A>^{2}+\frac{\beta^{\prime}}{T_{f}^{3}}<\Gamma_{1}, \Gamma_{1}>\right]<E, E>
$$

Recognizing that the coefficients in the brackets are all positive, to minimize $N_{e f f}$, we should make $E=0$.

Next, we find $F$ that minimizes $N_{\text {eff }}$. Similarly, $F$ only appears in the first and the fourth terms of the denominator in (12). After grouping together terms involving $F$ and discarding the rest, we have an object function $J(F)$ to minimize subject to the constraint $\left.<F, C_{p}\right\rangle=0$. The object function $J(F)$ is given by

$$
J(F)=\frac{4 P}{T_{f} N_{0}{ }^{2}}<F, F>+\frac{4 P}{T_{f}{ }^{2} N_{0}}<\Gamma_{2}, B><F, \frac{B *}{C} \Gamma_{1}>
$$

Using the method of Lagrange multiplier, the optimal $F$ can be found and is given by

$$
F=\frac{-N_{0}}{2 T_{f}}\left\langle\Gamma_{2}, B\right\rangle\left[g_{1} \frac{B *}{C} A+g_{2} \frac{B *}{C} B_{p}+\frac{B *}{C} D-g_{2} \frac{\left\langle B_{p}, B_{p}\right\rangle}{\left\langle C_{p}, C_{p}\right\rangle} C_{p}\right]
$$

Note $D$ in the above equation has to be replaced by the optimal one to be determined in the following.

Similarly for $D$ which only appears in the denominator, grouping together terms involving it and discarding the rest, we have the following object function to minimize to obtain the optimal $D$.

$$
\begin{aligned}
J(D) & =\frac{4 P}{T_{f} N_{0}^{2}}<F, F>+\frac{\beta^{\prime}}{T_{f}^{3}}<\Gamma_{2}, \Gamma_{2}><\Gamma_{1}, \Gamma_{1}> \\
& +\frac{4 P}{T_{f}^{2} N_{0}}<\Gamma_{2}, B><F, \frac{B *}{C} \Gamma_{1}>+\frac{P}{T_{f}^{3}}<\Gamma_{2}, B>^{2}<\Gamma_{1}, \Gamma_{1}>
\end{aligned}
$$

Minimizing $J(D)$ subject to the constraints $\langle D, A\rangle=0$ and $\left\langle D, B_{p}\right\rangle=0$ gives the optimal solution: $D=0$.

Plugging the optimal $D, E$, and $F$ into (13), we have the following equations of $\Gamma_{i}$ 's with $g_{i}$ 's to be optimized.

$$
\begin{aligned}
& \Gamma_{1}=g_{1} A+g_{2} B_{p} \\
& \Gamma_{2}=g_{3} A_{p}+g_{4} B \\
& \Gamma_{3}=g_{5} C_{p}-\frac{N_{0}}{2 T_{f}}<\Gamma_{2}, B>g_{1} \frac{B *}{C} A+g_{2} \frac{B *}{C} B_{p}-g_{2} \frac{<B_{p}, B_{p}>}{\left.<C_{p}, C_{p}\right\rangle} C_{p}
\end{aligned}
$$

Substituting the above equations into (12) gives $N_{\text {eff }}$ as a function of $g_{i}$ 's. The minimal $N_{e f f}$ and the optimal $g_{i}$ 's can be found by numerical search using math package softwares, which in turn gives the optimal pre-correlation filters $G_{i}$ 's.

\section{Bounds on the minimal $N_{e f f}$}

Although the optimal $\Gamma_{i}$ 's and hence the optimal $G_{i}$ 's can be found using the method described in the previous section, we are still interested in how close the optimal performance of a tracking loop is compared to the theoretical Cramer-Rao lower bound. Also, the set of pre-correlation filters $G_{i}$ 's found in the unmodulated and antipodally modulated cases seems another reasonable choices. We'd like to see how well the tracking loop performs using that set of filters.

The set of filters that minimizes $N_{\text {eff }}$ (and are also suggested by MLTE) in the unmodulated (10) and antipodally modulated (11) cases are given here again for clarity.

$$
\begin{aligned}
G_{1}^{*}(f) G_{4}(f) & =k_{1} \frac{1}{S_{n}(f)} \\
G_{2}^{*}(f) G_{5}(f) & =-k_{2} j 2 \pi f \tau_{m} \cdot \frac{1}{S_{n}(f)} \\
G_{3}^{*}(f) G_{6}(f) & =-k_{3} j 2 \pi f \tau_{m} \cdot \frac{1}{S_{n}(f)}
\end{aligned}
$$

Note that the optimality of these filters in their respective case is independent of SNR. Although this set of filters is not the optimal one in a closed-loop implementation for arbitrarily binary-modulated system, MLTE makes soft decision about $b_{i}$ and removes the modulation effectively hence the performance is expected to be close to the optimal one. Therefore, we have an upper bound for $N_{\text {eff }}$ as follows.

$$
\min N_{e f f} \leq\left.\bar{N}_{e f f}\right|_{\text {MLTE filters }}
$$

where $\bar{N}_{e f f}$ denotes an upper bound for $N_{\text {eff }}$ and the MLTE filters are those in (19). 


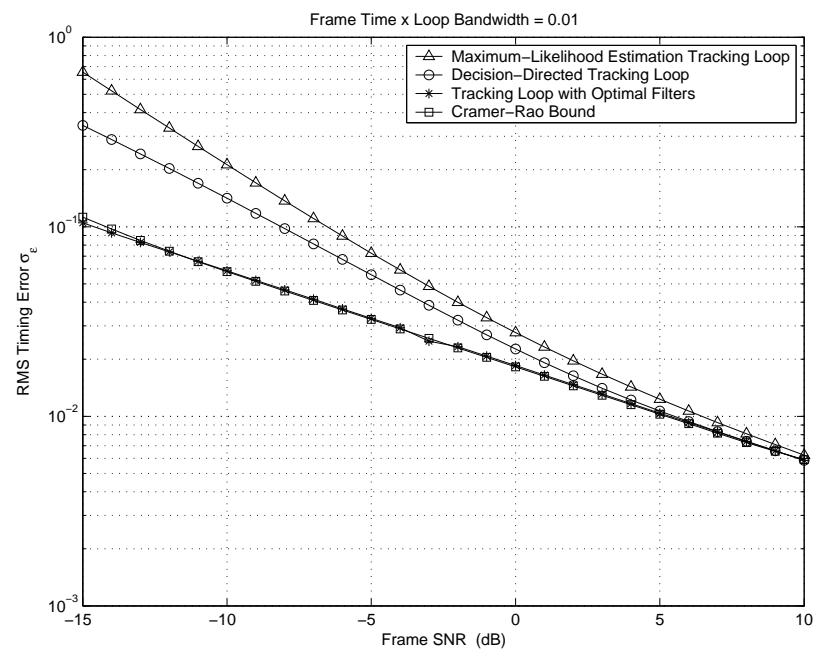

Fig. 3. Performance of different tracking loops and upper/lower bounds for optimal loop

For the lower bound on $N_{\text {eff }}$ we consider the CRLB of $\epsilon$. due to the space limitation, we omit the derivations here and only plot the results in the following section.

\section{Numerical Example}

Suppose Binary-PPM (non-antipodal) is employed over an AWGN channel and $s_{-1}(t)=s_{+1}\left(t-\delta \tau_{m}\right)$. Let $s_{+1}(t)=w_{2}(t)$ where $w_{2}(t)$ is the second derivative Gaussian waveform with unit power within one frame given by

$$
w_{2}(t)=\sqrt{\frac{8 T_{f}}{3 \tau_{m}}}\left[\begin{array}{lll}
1-4 \pi & \frac{t}{\tau_{m}} & 2
\end{array}\right] \exp \left[\begin{array}{lll}
-2 \pi & \frac{t}{\tau_{m}} & 2
\end{array}\right]
$$

and $\delta=0.45$ in the example.

The upper bound using MLTE filters (with $k_{1}=k_{2}=$ $k_{3}=1$ in (19)), the CRLB, and the minimal $N_{\text {eff }}$ using the optimal filters are plotted in Fig. 3. $\rho_{f}$ is the frame SNR defined by $\rho_{f} \triangleq P T_{f} / N_{0}$. Along with them, we also provide the performance curve of the decision-directed (DD) system which makes hard decision about $b_{i}$ and remove the modulation from the signal using this estimate.

We first notice that performance with optimal filters $G_{i}$ 's always lies between the upper and lower bounds, which demonstrates the validity of these bounds. Also, the indistinguishable performance curves of the optimal tracking loop and CRLB demonstrates its close-to-optimal performance. For low SNR region, the optimal loop outperforms DD and MLTE loops by a few dBs. At moderate to high SNR $\left(\rho_{f}>0 \mathrm{~dB}\right)$ where the linear theory is valid, the MLTE removes the modulation effectively by making correct decisions about $b_{i}$, hence the bounds are tight as expected. Also, the performance of the optimal loop and DD converge at high SNR as DD is designed for high SNR region. Usually, we require the rms timing jitter to be less than $10 \%$ of the pulse width. At this range, the optimal loop provides about $7 \mathrm{~dB}$ gain over traditional decisiondirected tracking loop.

We now look at the optimal filters $G_{i}$ 's and see how they differ from those suggested by the MLTE in (19). In con- trast to the cases of unmodulated and antipodally modulated systems in (19), the optimal values of $g_{i}$ 's (and hence the optimal $G_{i}$ 's) are functions of $\rho_{f}$. For example, at $\rho_{f}=5 \mathrm{~dB}$, the optimal $g_{i}$ 's are found to be

$$
\left\{g_{1}, g_{2}, g_{3}, g_{4}, g_{5}\right\}=\{1.00,-0.06,0.96,0.40,1.02\} .
$$

The optimal pre-correlation filters $G_{i}$ 's are then given by

$$
\begin{aligned}
& G_{1}^{*}(f) G_{4}(f)=1.00 \cdot \frac{1}{S_{n}(f)}+0.06 \cdot j 2 \pi f \frac{E_{m u}^{*}(f)}{S_{n}(f) E_{u}(f)} \\
& G_{2}^{*}(f) G_{5}(f)=-0.96 \cdot j 2 \pi f \tau_{m} \cdot \frac{1}{S_{n}(f)}+0.4 \cdot \frac{E_{m u}^{*}(f)}{S_{n}(f) E_{u}(f)} \\
& G_{3}^{*}(f) G_{6}(f)=-1.02 \cdot j 2 \pi f \tau_{m} \cdot \frac{1}{S_{n}(f)}-1.03 \cdot \frac{E_{m u}(f)}{S_{n}(f) E_{m}(f)}
\end{aligned}
$$

Note the filters in (23) are quite different from those suggested by MLTE in (19).

\section{Conclusion}

In this paper, the optimal performance of a tracking loop is investigated through pre-correlation filter optimization in an arbitrarily binary linearly modulated system. In particular, this optimal performance is compared with the CRLB and those of decision-directed tracking loop and a tracking loop suggested by MLTE. Numerical results show the optimal loop provides about $7 \mathrm{~dB}$ gain relative to decisiondirected tracking loop in the range of interest, and the performance is indistinguishable from the CRLB.

\section{REFERENCES}

[1] R. Sampaio-Neto and R. A. Scholtz, "Precorrelation Filter Design for Spread-Spectrum Code Tracking in Interference," IEEE $J S A C$, Vol. SAC-3, No. 5, pp. 662-675, Sep 1985.

[2] R. Sampaio-Neto, Spread Spectrum Code Tracking in the Presence of Interference, Ph.D. Dissertation, USC, LA, 1983.

[3] R. A. Scholtz, "Multiple Access with Time-Hopping Impulse Modulation," Proc. IEEE MILCOM'93, pp. 447-450, 1993.

[4] H. Meyr and G. Ascheid, Synchronization in Digital Communications, Wilet Inter-Science, 1990.

[5] R. De Gaudenzi and M. Luise, "Decision-Directed Coherent Delay-Lock Tracking Loop for DS-Spread-Spectrum Signals," IEEE Trans. Commun., Vol. COM-39, No. 5, pp. 758-79, May 1991. 